\title{
Relación del perfil de interés profesional y la carrera elegida por los estudiantes Universitarios
}

Enviado: 31 de octubre de 2021 / Aceptado: 23 de noviembre de 2021 / Publicado: 31 de diciembre de 2021

SIRLE ROSA HASEK DE BARBÚDEZ

Universidad Autónoma de Asunción (UAA), Paraguay sirhasek@gmail.com

(D) $0000-0001-6324-7201$

JOSÉ ANTONIO TORRES GONZÁLEZ

Universidad de Jaén, España jtorres@ujaen.es

0000-0001-6242-1191

\section{RESUMEN}

Esta investigación tuvo como objetivo relacionar el perfil de interés profesional y la carrera elegida por los estudiantes de la Universidad Nacional de Villarrica del Espíritu Santo. Se llevó a cabo en una Escuela de posgrado y ocho Facultades que ofrece carreras de grado en la Universidad Nacional de Villarrica del Espíritu Santo, con estudiantes del primer curso, la población participante fue de 503 estudiantes. Mediante un enfoque cuantitativo, con diseño no experimental, de alcance correlacional, se utilizó el método deductivo. Entre los principales resultados dentro del perfil de intereses profesionales los estudiantes de la carrera de Psicología, el 27 \% se identificaron con el código personal (SAE), Social, Artistico, Emprendedor, con el método del hexágono de Holland se evidencia que existe relación entre los códigos de las ocupaciones basados en la elección de la carrera y los perfiles de intereses profesionales, el $71 \%$ estu-

\section{ABSTRACT}

Relation of the profile of professional interest and the career chosen by the University students

This research aimed to relate the profile of professional interest and the career chosen by the students of the National University of Villarrica del Espiritu Santo. It was carried out in a Graduate School and eight Faculties that offers undergraduate degrees at the National University of Villarrica del Espiritu Santo, with students from the first year, the participating population was 503 students. Using a quantitative approach, with a non-experimental design, of correlational scope, the deductive method was used. Among the main results within the profile of professional interests, students of the Psychology degree, the $27 \%$ were identified with the personal code (SAE), Social, Artistic, Entrepreneur, with the Holland hexagon method it is evident that there is a relationship between the 
diaron el énfasis de Ciencias Sociales en la Educación Media, dicho énfasis se relaciona con la carrera elegida, el $41 \%$ de los códigos los las primeras letras en cada código son las mismas, por ende los intereses son consistentes y se relacionan entre sí.

Palabras Clave: Perfil de interés, Elección de carrera, Universidad. codes of occupations based on career choice and professional interest profiles, the , $71 \%$ studied the emphasis of Social Sciences in Secondary Education, this emphasis is related to the chosen career, $41 \%$ of the codes the first letters in each code are the same, therefore the interests are consistent and related each.

Keywords: Interest profile, Career choice, University.

\section{INTRODUCCIÓN}

El tema que se aborda en esta investigación trata de la relación del perfil de intereses profesionales y la carrera elegida por los estudiantes del primer curso de la Universidad Nacional Villarrica del Espíritu Santo. Al término de la Educación Media los estudiantes deben elegir su futura profesión, momento difícil que atraviesan los adolescentes, generando en ellos indecisiones y sobre todo una gran responsabilidad que influirá en su futuro. La elección de la carrera por parte de los estudiantes es una decisión personal de cada uno, de acuerdo a sus intereses y habilidades se inscriben en la carrera de su preferencia.

En la adolescencia cuando tienen que empezar a decidir cuál va a ser su futuro, a forjar su perfil e identidad profesional, esta tarea se vuelve difícil, pues tiene que decidirse en plena crisis puberal y psicológica: cambios corporales, inseguridad, deseos de independencia, cambios continuos de intereses, etc., que van a complicar la decisión, ya que el sujeto está inmaduro no sólo vocacionalmente sino en el ámbito de toda su personalidad. A pesar de todo, el joven debe decidirse, intentando que la elección le permita alcanzar un nivel óptimo de satisfacción individual (autorrealización), de adaptación y de compromiso social (Aguirre, 1994). Es importante que los estudiantes del primer curso de la Universidad Nacional de Villarrica del Espíritu Santo conozcan la relación del perfil de interés profesional y la carreara elegida, de manera a conocerse mejor a sí mismos, en cuanto a sus intereses, habilidades, aptitudes y vocaciones, para que se sientan identificados con sus futuras profesiones.

Por otra parte, el resultado obtenido en ésta investigación permite explorar las carreras de acuerdo a sus aspiraciones ocupacionales, beneficioso para los estudiantes, como también a la comunidad educativa porque tienen la posibilidad de conocer a través de análisis gráficos la relación entre el perfil de interés profesional y la carrera elegida por los estudiantes 
del primer curso insertados en la búsqueda de sus profesiones con una visión diferente en la sociedad actual, además ayuda a conocer las capacidades y competencias de la formación de los estudiantes para tomar decisiones propias en el desarrollo integral y la identificación de la profesión deseada de modo a sentirse seguros de sí mismos, porque con ella se logra que los alumnos se conozcan a sí mismos y los resultados obtenidos sirven para que la comunidad educativa identifique la realidad de los estudiantes, para apoyar el conocimiento del perfil de intereses profesional, así conocerse a sí mismo al iniciar la carrera profesional, según Holland, 1985 (citado en Holland, Frtzsche y Powel, 2005b) conduce a las definiciones tipológicas y a la teoría que subyace al esquema de elección y clasificación ocupacionales, así como también las siete suposiciones que subyacen a la tipología:

- Puede categorizarse a la mayoría de las personas como uno de los seis tipos de personalidad a los que se ha dado los nombres: Realista (R), Investigador (I), Artístico (A), Social (S), Emprendedor (E), o Convencional (C).

- Existen seis tipos de ambientes: Realista (R), Investigador (I), Artístico (A), Social (S), Emprendedor (E), o Convencional (C).

- Las personas buscan el tipo de ambiente que le permite ejercer sus habilidades y capacidades, expresar sus actitudes y valores, enfrentarse a problemas y roles compatibles.

- La conducta de una persona se determina por la interacción entre su personalidad y las características del ambiente.

- El grado de congruencia o concordancia entre una persona o una ocupación (ambiente) puede calcularse por medio de un modelo hexagonal.

- El grado de consistencia dentro de una persona o ambiente también se define utilizando el modelo hexagonal.

- El grado de diferenciación de una persona o ambiente modifica las predicciones que pueden hacerse a partir del perfil SDS (Búsqueda Autodirigida) de la persona, el código ocupacional o de la interacción entre ambos. La Búsqueda Autodirigida SDS, es el resultado de una teoría de elección ocupacional, se ha basado en la teoría de los tipos de personalidad y modelos ambientales.

Además, estos perfiles son compatibles con el modelo de Holland $(1985,1997)$ de los tipos de personalidad y condiciones de trabajo. Se utilizan seis categorías de interés para describir los ambientes laborales de las profesiones: Realista, investigadora, artística, social, emprendedora y convencional (Holland, Frtzsche y Powel, 2005a). También menciona a Holland (1983) quien propone un enfoque tipológico en el cual considera, en principio, un modelo estructural 
basado en los planteamientos de Parsons, aunque más elaborado y actualizado que el esquema clásico de rasgos y factores. Propone una serie de principios que considera fundamentales en un enfoque de tipo estructural e interactivo:

La elección de una vocación es expresión de la personalidad; los inventarios de intereses son inventarios de personalidad; los estereotipos vocacionales tienen significados psicológicos y sociológicos confiables e importados; los miembros de una profesión tienen personalidades similares e historias parecidas de desarrollo personal; las personas de un grupo ocupacional tienen personalidades similares, responderán a muchas situaciones y problemas de manera análoga y crean medios interpersonales característicos; la satisfacción, estabilidad y logro ocupacional dependerá de la congruencia entre la personalidad de uno y el ambiente de trabajo.

Para este enfoque los intereses vocacionales proceden de la personalidad, las personas suelen tener una idea fija sobre las ocupaciones, cada profesión atrae y mantiene a individuos que poseen personalidades afines y un individuo se encuentra más satisfecho en aquellos trabajos o profesiones que mejor se ajustan a esa persona, cada quien posee intereses diferentes, los tipos de intereses según Holland, et al. (2005b) son:

$\mathrm{Al}$ realista le gustan las actividades y ocupaciones que involucran, uso de máquinas herramientas y cosas, valora, reconocimiento monetario por los logros observables, la honestidad, el sentido común, se ve a sí mismo como, práctico, conservador y con más habilidades manuales y mecánicas que sociales, otros lo ven como, humilde, franco, autosuficiente, persistente, evita, interacción con otras personas.

Al investigador le gustan las actividades y ocupaciones que involucran, exploración y entendimiento de cosas y eventos, valora, conocimiento, aprendizaje, logro, independencia, se ve a sí mismo como analítico, inteligente, escéptico y con más destrezas académicas que sociales, otros lo ven como, inteligente, introvertido, académico, independiente, evita, tener que convencer a otros o venderles cosas.

$\mathrm{Al}$ artístico le gustan las actividades y ocupaciones que involucran leer libros, actividades musicales o artísticas, escribir, valora ideas creativas, auto expresión, belleza, se ve a sí mismo como, receptivo a las experiencias, imaginativo, intelectual y con más destrezas creativas que administrativas o de oficina, otros lo ven como, poco común, desordenado, creativo, sensible, evita rutinas y reglas.

Al social le gustan las actividades y ocupaciones que involucran, ayudar, enseñar, aconsejar o servir a otros, valora, servicio social, justicia, comprensión, se ve a sí mismo como, 
empático, paciente y con más destrezas sociales que mecánicas, otros lo ven como, servicial agradable, extravertido, paciente, evita actividades mecánicas y técnicas.

Al emprendedor le gusta las actividades y ocupaciones que involucran, persuadir a otros, valora, éxito financiero y social, lealtad, tomar riesgos, responsabilidad, se ve a sí mismo como, seguro de sí mismo y con más habilidad de ventas y de persuasión que científica, otros lo ven como, enérgico, extravertido, astuto y ambicioso, evita temas científicos, intelectuales o complicados.

Al convencional, le gusta las actividades y ocupaciones que involucran, seguir rutinas ordenadas, satisfacer estándares claros, valora, precisión, ganar dinero, economizar, poder en asuntos de negocios o sociales, se ve a sí mismo como, con más destrezas técnicas en comercio o producción que habilidades artísticas, concienzudo, practico, otros lo ven como, cuidadoso, seguidor de reglas, eficiente, ordenado, evita trabajo que no tiene instrucciones claras.

La elección vocacional, de oficio o carrera profesional, es el resultado de un conjunto de factores y de condicionantes sociales, así como de esfuerzos, expectativas e interés personal, que concurren a lo largo de un proceso que se desarrolló en el tiempo y en los lugares en que transcurre la vida de quienes toman dicha decisión (Rodríguez, Sánchez y Lavajos, 2017). Los valores actuales trasmiten erróneamente, la importancia de ganar dinero o tener éxito social y poder antes que o por encima de las preferencias vocacionales profesionales a la hora de realizar la elección (González, 2004).

Según Riart (2001), las elecciones vienen determinadas por este factor seguido de la vocación, por lo que muchas personas están desubicadas entre sus aspiraciones personales y sus logros profesionales. La preferencia vocacional supera en extensión al concepto de interés vocacional que es tentativo. Tanto la expresión de deseo o atracción hacia determinadas actividades profesionales, como la proyección de uno mismo respecto a la posibilidad de llegar a desempeñarlas, permiten predecir la conducta vocacional futura y ayudar al desenvolvimiento vocacional y profesional de la persona (Cepero, 2009).

En la elección vocacional se ponen en juego tanto el ideal del yo como las idealizaciones, uno de los efectos más observados en nuestra práctica es la configuración de procesos de inhibición, soñar, imaginar optar por algo que se presenta idealizado y por ende, inalcanzable, es la mejor defensa para no activar (Rascovan, 2016).

Es de suma importancia que la orientación vocacional se dé desde los primeros años de escolaridad, y no se limite al momento de la elección de la carrera, al final de la educación media. Es una etapa en la que necesitan orientación, información adecuada y acompañamiento 
específico frente a sus dudas, inquietudes y expectativas sobre su proyecto de vida, es por ello que se plantea los programas de Orientación Vocacional para estudiantes de nivel secundaria (Ministerio de Educación, 2011).

La orientación vocacional es un proceso complejo que se desarrolla desde la infancia, bajo la influencia de varios aspectos internos y externos (Ramos, Bravo, Ravello, Chávez, Sabaduche, 2013).

El Ministerio de Educación y Ciencias MEC, asume una perspectiva pedagógica de la política educativa, lo cual significa que sus decisiones y acciones como Cartera de Estado no deben ser entendidas como un determinismo estatista, sino como un planteamiento y replanteamiento participativo continuo del sentido del Sistema Educativo; la política educativa que se propone desde el Gobierno Nacional dota de direccionalidad conceptual, técnica, ética y política al quehacer dinámico que se da en las relaciones educativas cotidianas que hacen a la formación de las personas como seres originales y protagonistas activos en la construcción del contexto sociocultural del Paraguay contemporáneo (Ministerio de Educación y Cultura. MEC, 2011).

En la investigación se plantea el siguiente objetivo general: Relacionar el perfil de intereses profesionales y la carrera elegida por los estudiantes del primer curso de la Universidad Nacional Villarrica del Espíritu Santo, año 2018 y los objetivos específicos son: Analizar el perfil de intereses profesionales de los estudiantes del primer curso, identificar las careras elegidas por los estudiantes y relacionar el perfil de intereses profesionales y la elección de la carrera de los estudiantes del primer curso con la carrera elegida por los estudiantes.

En ésta investigación se plantean las siguientes hipótesis: El perfil de intereses profesionales se relaciona con la carrera elegida por los estudiantes del primer curso de la Universidad Nacional Villarrica del Espíritu Santo.

\section{MATERIAL Y MÉTODO}

En esta investigación se utilizó el enfoque cuantitativo, con alcance correlacional.

La investigación se ha llevado a cabo en todas las Facultades y una Escuela dependiente de la Universidad Nacional Villarrica del Espíritu Santo, todas ellas entregan los títulos de grado al término de las carreras que ofrecen. La población en el momento de la investigación correspondió a 571 estudiantes, participaron 503 estudiantes del primer curso debido a la ausencia de 68 en el momento de aplicación de los instrumentos. 
Tabla 1. Facultades y Escuelas de la Univ ersidad Nacional Villarrica del Espíritu Sant o. Fuente: Autoría Propia

\begin{tabular}{|c|c|c|c|}
\hline FACULTADES & CARRERAS & $\begin{array}{c}\text { TOTAL DE } \\
\text { ESTUDIANTES }\end{array}$ & $\begin{array}{l}\text { POBLACIÓN } \\
\text { PARTICIPANTE }\end{array}$ \\
\hline \multirow[t]{3}{*}{ Facultad de Ciencias de la Salud } & Lic. Nutrición & 22 & 19 \\
\hline & Lic. Psicología & 18 & 18 \\
\hline & Lic. En enf ermería & 42 & 37 \\
\hline \multirow[t]{2}{*}{ Facultad de Ciencias Agr arias } & Ing. En Agr oindustria & 60 & 59 \\
\hline & Ing. En Zoot ecnia & 18 & 12 \\
\hline \multirow[t]{3}{*}{ Facultad de Filosofía y Ciencias Humanas } & Lic. Ciencias de la educación & 32 & 24 \\
\hline & Lic. Inglés & 18 & 13 \\
\hline & Lic. Comunicación & 21 & 21 \\
\hline \multirow[t]{2}{*}{ Facultad de Ingeniería y Ar quitectura } & Ing. Ambient al & 43 & 40 \\
\hline & Lic. Ar quitectura & 45 & 39 \\
\hline Facultad de Química, Física y Mat emáticas & Ing. Química & 32 & 26 \\
\hline \multirow[t]{2}{*}{ Facultad Politécnica } & Ing. En análisis de sist emas & 45 & 37 \\
\hline & Lic. En electricidad & 40 & 33 \\
\hline \multirow[t]{2}{*}{ Facultad de Ciencias Económicas } & Administr ación de empr esa & 28 & 27 \\
\hline & Contaduría pública & 34 & 34 \\
\hline $\begin{array}{l}\text { Facultad de Ciencias Jurídicas, Políticas y Socia- } \\
\text { les. }\end{array}$ & Derecho & 40 & 33 \\
\hline Escuela de Gastr onomía, Hotelería y T urismo & Lic. En Gastr onomía & 33 & 31 \\
\hline TOTAL & & 571 & 503 \\
\hline
\end{tabular}

Nota: La Escuela de Art e y Cultur a no p articipa de est a inv estigación por que se encontr aba en proceso de selec ción de estudiant es para el ingr eso

Se aplicó el cuestionario de la Búsqueda Autodirigida SDS Forma R, para explorar los intereses y las aspiraciones vocacionales, ayuda a la interpretación de los perfiles de una persona basándose en los principios generales de la medición y de la Psicometría, se utilizaron instrumentos consistentes en tres cuadernillos: Cuaderno de autoevaluación, Explorador de carreras y ocupaciones, Tu carrera y Vocación. En cuanto a la confiabilidad, los coeficientes de consistencia interna (fórmula 20 de Kuder Richardson: K-20: Kuder y Richardson, 1937) para una muestra de estudiantes universitarios fueron de 53 a.85 (X=.74) para mujeres y de .63 a $88(\mathrm{X}=.78)$ para varones (Gottfredson y Holland, 1975b). Con pocas excepciones, la $1^{\circ}$ edición original de las escalas SDS tuvo un grado moderado de consistencia interna. Las correlaciones para la escala de resumen eran de 59 a 95 (Holland et al, 2005b). 
La validez de contenido de todos los reactivos y escalas dentro del SDS es aparente en el contenido y formato de los mismos. Todos los reactivos se expresan de manera positiva y directa de modo que sólo se requiere un mínimo de interpretación. Además cada escala se compone de reactivos que claramente se relacionan con esa escala específica. El contenido es consistente con conocimientos ocupacionales bien establecidos. (Holland, et al., 2005a).

Antes de la aplicación del instrumento se solicitaran los datos a los estudiantes para confirmar el énfasis de la Educación Media realizado y la carrera que está cursando.

Se utilizaron programas de software Microsoft Excel y Word, se realizó la tabulación de datos a través de tablas de frecuencias que permitió recontarlos, resumirlos, posibilitar la interpretación, codificación, realizar gráficos en barra y el logro de conclusiones a través de los resultados obtenidos en la aplicación del SDS Búsqueda Autodirigida. Para establecer la relación del perfil profesional y la carrera elegida se utilizó el método del modelo hexagonal de Holland, entre el código de la elección ocupacional y el código personal resultante, calculado de la siguiente manera para posteriormente computarlo en porcentajes.

\section{REFERENCIA:}

Las primer as letr as se oponen una a otr a en el hexágono .

Las primer as letr as no son ady acentes ni opuest as una a otr a en el hexágono.

Las prime as letr as son ady acentes una a la otr a en el hexágono .

Las primer as letr as en cada código son las mismas.

A mayor puntuación may or relación
Modelo Hexagonal de Holland

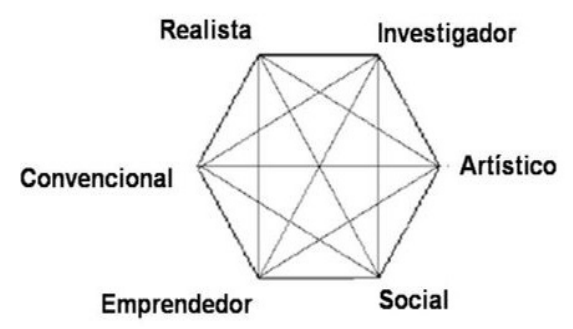

Fuente. (Holland, et al., 2005b)

\section{RESULTADOS}

El perfil de intereses profesionales ha sido el conjunto de capacidades y competencias que identifican la formación de una persona para asumir en condiciones óptimas las responsabilidades propias del desarrollo de funciones y tareas de una determinada profesión Hawes y Corvalán, (2005); para describirla se identificaron los códigos personales, los tipos de personalidad, (según citó Holland et al., 2005b) y también se buscó las profesiones en el explorador de carreras, se obtuvieron los siguientes resultados: 
Gráfico 1. Códigos personales de los estudiantes del primer curso de la Univ ersidad Nacional de Villarrica del Espíritu Sant o.

Fuente. Elabor ación pr opia

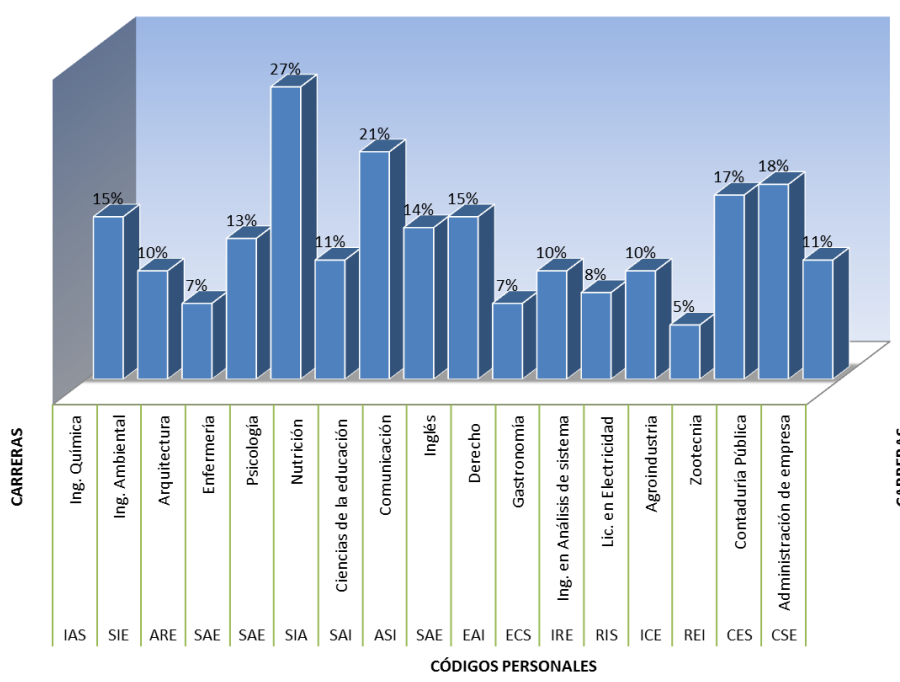

Gráfico 2 . Síntesis de los tipos de personalidad de los estudiant es del primer curso de la Univ ersidad Nacional de Villarrica del Espíritu Sant o. Fuente. Elaboración pr opia

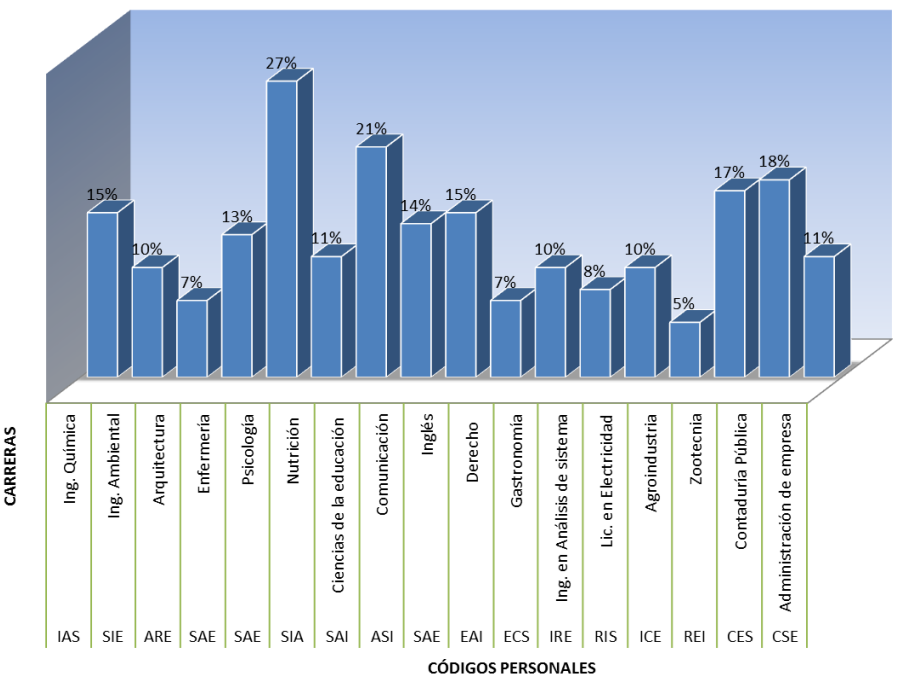

La identificación de los códigos profesionales de tres letras proveyó descripciones de caracteristicas de una carrera, basada en las habilidades, las ocupaciones y las actividades que le gusta realizar, facilitó la obtención del código Holland, mediante la cual se confirma que el 27 \% de los estudiantes de la carrera de Psicología posee el código personal correspondiente a lo Social, Artistico, Emprendedor (SAE), alcanzando el porcentaje más elevado, en cuanto a la primera letra del código Social la misma responde a ocupaciones sociales que se detalla según Holland, Frtzsche y Powel (2005c) en el explorador de carreras y ocupaciones, que corresponde a algunas profesiones como por ejemplo: Orientador vocacional, bibliotecario, psicólogo, enfermería, oficial de policía, notario público entre otros.

Recordemos que los tipos de personalidad según Holland son Realista (R), Investigador (I), Artístico (A), Social (S), Emprendedor (E), y Convencional (C), este resultado arrojó el parentesco según características que corresponde a los tipos de personalidad. Se evidenció que de los 503 estudiantes del primer curso de la Universidad Nacional de Villarrica de las carreras de Enfermería, Psicología, Nutrición, Ciencias de la Educación, Derecho, Contaduría pública, Administración de Empresas, Inglés y Zootecnia el $41 \%$ se identificó con el tipo de personalidad correspondiente a lo Social, según Holland et al. (2005c), tienen habilidades y talento social, les gusta ayudar, enseñar, aconsejar o servir a otros. Son bondadosos, comprensivos, cooperadores, generosos, pacientes, responsables, serviciales entre otros. 
Gráfico 3. Relación del énf asis de la Educación Media y la carr era elegida por los estudiant es de la Univ ersidad Nacional Villarrica del Espíritu Sant o. Fuente. Elabor ación pr opia

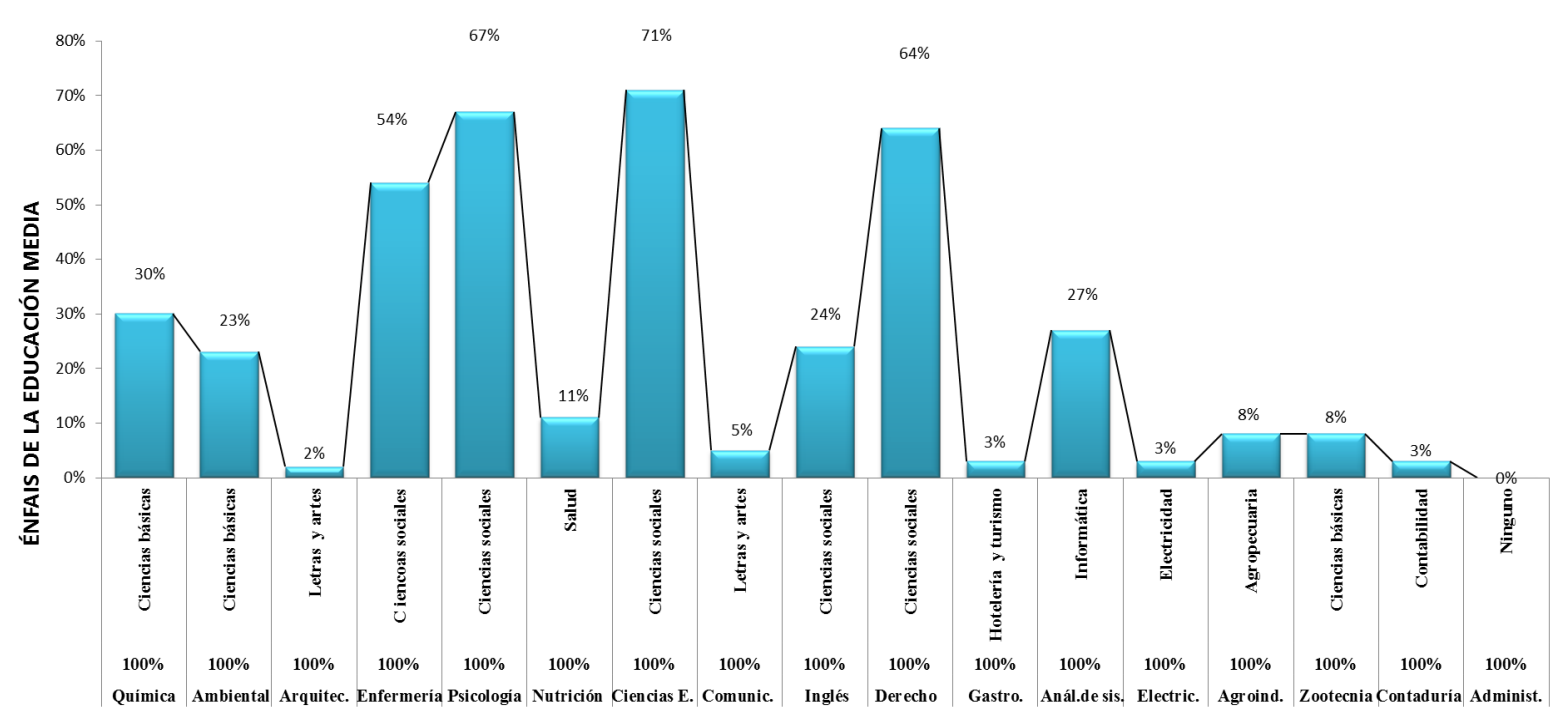

CARRERAS ELEGIDAS

Gráfico 4. Perfil de in erés profesional y carr era elegida. Fuente. Elabor ación pr opia

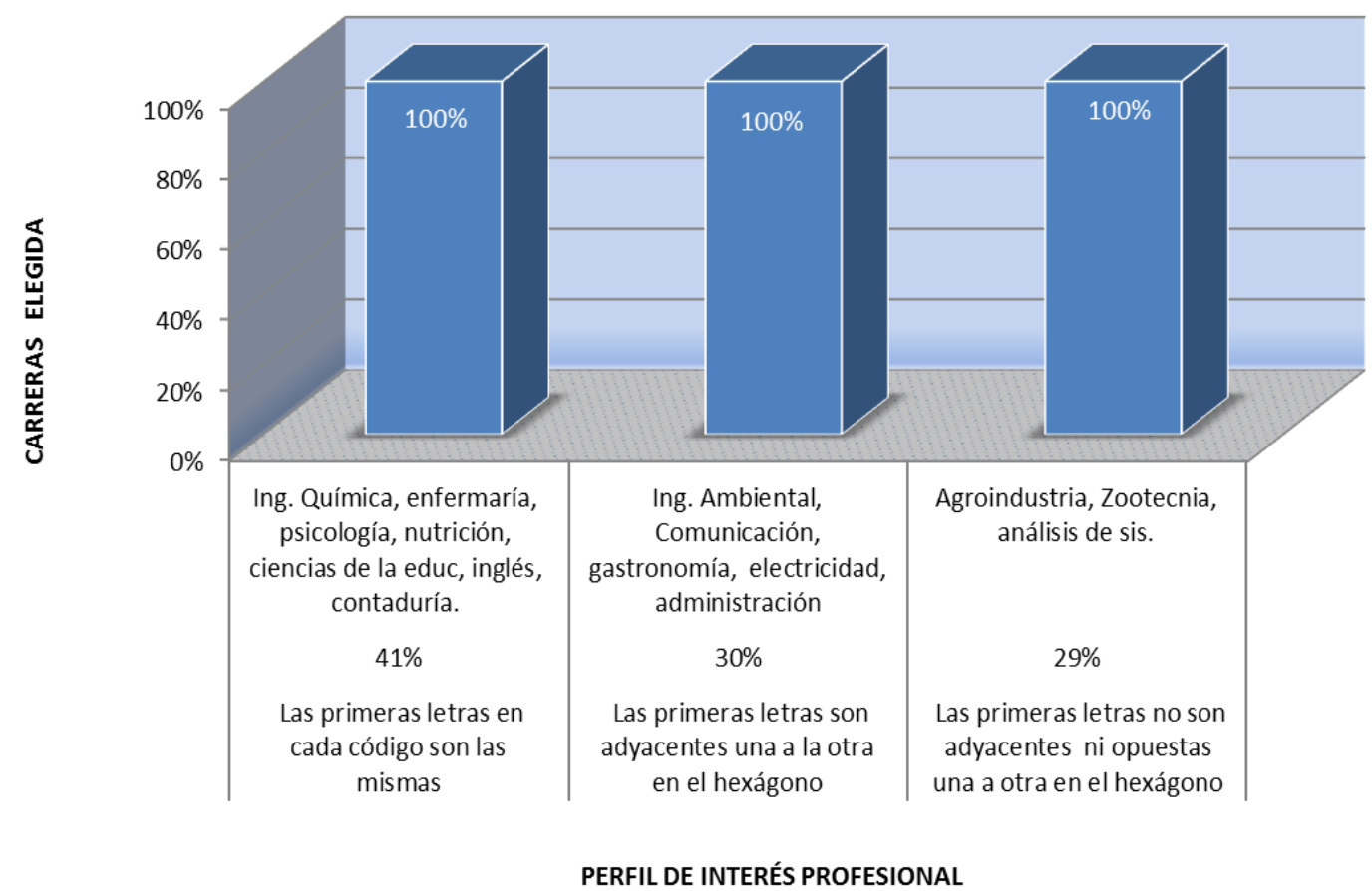

El Ministerio de Educación y Ciencias ofrece a los estudiantes de la Educación Media la posibilidad de elegir el énfasis que más le guste a fin de que sirva como base para la formación universitaria a fines a la carrera elegida. La carrera de Ciencias de la Educación alcanzando el 
porcentaje más elevado, 71 \% estudiaron el énfasis de Ciencias Sociales en la Educación Media dicho énfasis se relacionó con la carrera elegida, la misma responde a ocupaciones Sociales que se detalló según Holland et al. (2005c), en el explorador de carreras que correspondió a algunas profesiones como por ejemplo: Profesor de escuela secundaria, Profesor de escuela primaria, Profesor de Educación para adultos, Profesor de Educación Indígena, Profesor Universitario, Director de Escuelas, Orientador vocacional, Instructor, Bibliotecario entre otros.

Seguido del $67 \%$ de los estudiantes de la carrera de Psicología terminaron su Educación Media en el énfasis en Ciencias Sociales la misma respondió a ocupaciones sociales que se detalló en el explorador de carreras que correspondió a algunas profesiones como por ejemplo: Psicólogo, Psicopedagogo, Psicólogo clínico, psicólogo social, psicólogo consejero, Psicoterapeuta, psicólogo infantil, Orientador vocacional, entre otros, por ende se relacionó entre sí, así como también los demás énfasis y carreras guardaron relación de menor porcentaje.

Se pudo constatar, la relación entre los códigos de las ocupaciones basados en la elección de la carrera de los 503 estudiantes de las ocho facultades y una escuela de posgrado que ofrecen títulos de grado, abarcando así 17 carreras de las cuales se encuentran en el primer lugar las carreras de Ingenieria Química, Enfermería, Nutrición, Ciencias de la Educación, Inglés y Contaduría Pública donde se evidenció que en un 41 \% las primeras letras en cada código son las mismas por ende los intereses son más consistentes o compatibles y se relacionan entre sí, los estudiantes de las carreras de Ingeniería Ambiental, Comunicación, Gastronomía, Electricidad, y Administración han demostrado que en un $30 \%$, las primeras letras del código son adyacentes una a la otra en el hexágono, esto implica relativamente que es menos compatible pero está relacionada. Además los estudiantes de las carreras de Agroindustria, Zootecnia y Análisis de Sistemas han demostrado en un 29 \% que las primeras letras de sus códigos no son adyacentes ni opuestas una a otra en el hexágono por ende se hallan en una posición menos armónica.

\section{DISCUSIÓN}

En esta investigación se relacionó el perfil de intereses profesionales y la carrera elegida de los estudiantes del primer curso de la Universidad Nacional Villarrica del Espíritu Santo, los estudiantes en su mayoria se identifican con el código (SAE) Social, Artístico y Emprendedor, sobresaliendo así en lo Social (S), donde las aptitudes interpersonales, destrezas para enseñar, tratar o sanar a otros con sus principales prioridades dentro de las ocupaciones, según Holland et al. (2005c), el código personal sintetiza el parecido de una persona con los tipos de personalidad, por ende se pudo constatar que la mayoría de los estudiantes se identifican 
con el tipo de personalidad Social (S), coincidiendo de esta manera con la teoría de Holland que pronostica el resultado de la combinación, en cuanto a la relación del perfil de interés profesional y la carrera elegida el $41 \%$ de los estudiantes demuestran que las primeras letras en cada código son las mismas por ende los intereses son más consistentes o compatibles y se relacionan entre sí, en la carrera de Ingeniería Química, Enfermería, Nutrición, Ciencias de la Educación, Inglés y Contaduría Pública.

\section{CONCLUSIÓN}

Este trabajo ha contribuido en brindar un espacio para que los estudiantes puedan conocer sus habilidades, las ocupaciones que le interesan, las actividades que les gusta realizar, en fin a conocerse a sí mismo. Además brinda a la Escuela y a las Facultades informaciones muy importantes que ayuda a plantear acciones en base a los resultados obtenidos, contribuye a conocer la realidad educativa de los jóvenes que cursan el primer curso de la Universidad Nacional de Villarrica del Espíritu Santo.

\section{REFERENCIAS BIBLIOGRÁFICAS}

Aguirre, B. A. (1994). Psicología de la adolescencia. Editorial Boixareu Universitaria, marcombo.

Cepero, G. A. (2009). Las Preferencias Profesionales y Vocacionales del Alumnado de Secundaria y Formación Profesional Especifica. (Tesis Doctoral, Universidad de Granada). https://hera.ugr.es/ tesisugr/18751362.pdf

González, M. V. (2004). La orientación profesional y curriculum Universitario. Una estrategia educativa para el desarrollo profesional y responsable. Alertes. Psicopedagogía.

Holland, J. L., Fritzsche, B. A. y Powell A. B. (2005a). SDS Búsqueda Autodirigida. Guía para el usuario profesional. Manual Moderno.

Holland, J. L., Fritzsche, B. A. y Powell A. B. (2005b). SDS Búsqueda Autodirigida. Manual técnico. Manual Moderno.

Holland, J. L., Fritzsche, B. A. y Powell A. B. (2005c). SDS Búsqueda Autodirigida. Explorador de carreras y ocupaciones. Forma R. Manual Moderno.

Riart, J. (2001). La orientación vocacional profesional y ocupacional en el 2 ciclo de la ESSO. Recuperado de https://educaweb.com 
Rodríguez, M. M., Sánchez R. E. y Labajos M. M. (2017). Vocación ocupacional y género en estudiantes universitarios de Ciencias de la Salud. Revista Latinoamericana de Ciencias de la Salud Sociales, Niñez y fuventud, 15(1), pp. 345-356. https://www.redalyc.org/pdf/773/77349627022.pdf

Rascovan, S. (2016). La orientación vocacional como experiencia subjetivante. Paidós.

Ramos, M. T. Bravo, C., Ravello, Chávez, M., Sabaduche, L. (2013). Orientación Vocacional. Lima. M \& J GRAF E.I.R.L.

Ministerio de Educación. (2011). Tutoría y Orientación Educativa. Orientación Vocacional. Cartilla para tutores. Primera Edición Lima.

Ministerio de Educación y Cultura (2014). Actualización Curricular del Bachillerato Científico de la Educación Media - Plan Específico: Énfasis en Ciencias Básicas y Tecnología. MEC. 